\title{
Control under Clock Offsets and Actuator Saturation
}

\author{
Masashi Wakaiki, Kunihisa Okano, and João P. Hespanha
}

\begin{abstract}
This paper studies the stability analysis and the stabilization problem for systems with asynchronous sensors and controllers, and actuators subject to saturation. We consider a system with parameter uncertainty caused by clock offsets. By using a polytopic overapproximation, we investigate how large clock offsets affect stability. In addition, we employ a sector characterization approach to address actuator saturation. We see from a numerical example that the range of allowable clock offset bounds drops if the saturation limit becomes smaller than a certain value.
\end{abstract}

\section{INTRODUCTION}

The effects of networked-induced delays and variable sampling intervals have been actively studied, as surveyed in, e.g., [1]-[3]. A solution to compensate such uncertainties in the time domain is that the sensor sends the measurement together with its time-stamp [4], [5]. Time-stamping allows the controller to estimate the plant's state under the assumption that the clock of the controller is synchronized that of the sensor. However, perfect clock synchronization is fundamentally impossible [4], [6]. We therefore need to address the existence of a clock offset between the sensor and the controller, as it may degrade the performance of the closed-loop system and can even destabilize the system. Imperfect timing on control has been studied for contentioustime systems in [7], [8] and for the discrete-time systems in [9]. In this work, we consider sampled-data systems, where the plant's state is estimated at each control-updating instant.

The main objective of this paper is to determine how large a clock offset can be allowed until it compromises stability. In our previous work [10], the stabilization problem of systems with constant clock offsets has been studied. This paper considers more practical situations in the following two senses: We study systems with time-varying clock offsets that take values in a bounded interval. Clock offsets are time varying in practical applications because the oscillator in a clock may be affected by its environment such as temperature and humidity. Our recent work [11] has also investigated systems with time-varying clock offsets and proposed a stabilization method with causal controllers, based on the analysis of data rate limitations in quantized control. Here we use controllers consisting of a linear estimator and a

This material is based upon work supported by the National Science Foundation under Grant No. CNS-1329650. M. Wakaiki acknowledges The Kyoto University Foundation and Murata Overseas Scholarship Foundation for the support of this work and K. Okano is supported by JSPS Postdoctoral Fellowships for Research Abroad.

The authors are with the Center for Control, Dynamical-systems and Computation (CCDC), University of California, Santa Barbara, CA 93106-9560 USA (e-mail: masashiwakaikidece.ucsb.edu; kokanodece.ucsb.edu; hespanhadece.ucsb.edu). static gain, and study stability analysis and the design of stabilizing gains from the perspective of robust control.

The second contribution is to study the stability of systems with actuator saturation in addition to time-varying clock offsets. Actuator saturation is an ubiquitous nonlinearity in engineering applications and could make feedback systems unstable. In [12], [13], control problems with saturation have been studied in the context of networked control systems, in particular, with quantization and variable delay. However, relatively little work has been done towards investigating how actuator saturation affects the range of clock offsets that would be allowed for stability.

First we analyze the effect of clock offsets on the stability of a closed-loop system with ideal actuators. The networked control system we consider is modeled as a discrete-time linear parameter-varying system. In the resulting discretized system, the variable clock offset appears in an exponential form. We therefore use the overapproximation technique in [2], [14], [15] to embed the original model into a larger class of polytopic models with a norm-bounded additive uncertainty. Using this overapproximated system, we provide a sufficient condition for stability in terms of linear matrix inequalities (LMIs). From this condition, two design methods of static stabilizing feedback gains are also presented.

Next we extend the first result to the analysis of local stability for systems with actuator saturation as well as clock offsets. In order to address a saturation nonlinearity, we use the sector characterization proposed in [16], [17], [18, Chapter 3], which can be regarded as an overapproximation technique for actuator saturation. We observe from a numerical study that the allowable offset range decreases sharply when the saturation limit is smaller than a certain value.

This paper is organized as follows. The next section gives the closed-loop system we consider and presents the problem formulation. In Section III, we study the stability analysis and the design of feedback gains for systems using timevarying clock offsets with results in [2], [14]. Section IV is devoted to extend the results in Section III to the regional stability analysis of systems with clock offsets and actuator saturation. Finally, concluding remarks are given in Section V.

Notation: Let $\mathbb{Z}_{+}$be the set of non-negative integers. For a vector $v$, we denote by $\|v\|$ the Euclidean norm of $v$. For a matrix $M$, we denote by $\|M\|$ and $\operatorname{trace}(M)$ the Euclideaninduced norm and the trace of $M$, respectively. Also for square matrices $M_{1}, \ldots, M_{n}, \operatorname{diag}\left(M_{1}, \ldots, M_{n}\right)$ means the block diagonal matrix such that the main diagonal blocks starting in the upper left corner are $M_{1}, \ldots, M_{n}$ and the off-diagonal blocks are zero matrices. 


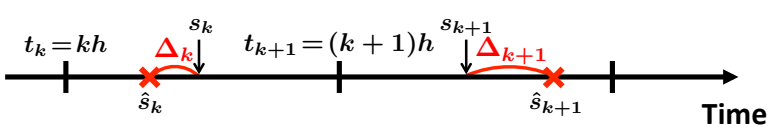

Fig. 1: Sampling instants $s_{k}$, reported time-stamps $\hat{s}_{k}$, and updating instants $t_{k}$ of the zero-order hold

\section{Problem Statement}

Consider the following plant:

$$
\dot{x}(t)=A x(t)+B u(t),
$$

where $x(t) \in \mathbb{R}^{n}$ and $u(t) \in \mathbb{R}^{m}$ are the state and the input of the plant, respectively. To simplify the system representation, we introduce the following assumption:

Assumption 2.1: The matrix $A$ is invertible.

Let $s_{0}, s_{1}, \ldots$ be sampling instants. The sensor observes the state $x\left(s_{k}\right)$ and sends it to the controller together with its time-stamp. However, since the sensor and the controller share no global clock, the time-stamp typically includes an unknown offset with respect to the controller clock. In this paper, we assume that the offset is time-varying and bounded, that is, for each $k \in \mathbb{Z}_{+}$the sampling instant $s_{k}$ and the time-stamp $\hat{s}_{k}$ reported by the sensor have the following relationship: $s_{k}=\hat{s}_{k}-\Delta_{k}$ for some unknown clock offset $\Delta_{k} \in[\underline{\Delta}, \bar{\Delta}]$.

Let $h>0$ be the update period of the control signal $u(t)$. The control signal $u(t)$ is piecewise constant and updated at times $t_{k}=k h\left(k \in \mathbb{Z}_{+}\right)$with values $u_{k}: u(t)=u_{k}$ for $t \in$ $\left[t_{k}, t_{k+1}\right)$. While the control input is updated periodically, the true sampling times $s_{k}$ and the reported sampling times $\hat{s}_{k}$ may not be periodic. We assume that both $s_{k}$ and $\hat{s}_{k}$ do not fall behind $t_{k+1}$ by more than $h$, and that the controller side receives the state measurement $x\left(s_{k}\right)$ and the time-stamp $\hat{s}_{k}$ transmitted from the sensor by the next control-updating instant $t_{k+1}$. This assumption is formally stated as follows.

Assumption 2.2: For $k \in \mathbb{Z}_{+}, s_{k}, \hat{s}_{k} \in\left[t_{k}, t_{k+1}\right)$. Furthermore, $x\left(s_{k}\right)$ and $\hat{s}_{k}$ are available to the controller by time $t=t_{k+1}$.

Fig. 1 shows the timing diagram of the sampling instants $s_{k}$, the reported time-stamps $\hat{s}_{k}$, and updating instants $t_{k}$ of the control inputs.

The controller estimates the state of the plant at time $t=$ $t_{k+1}$ using the following dynamics after receiving the data $x\left(s_{k}\right)$ and $\hat{s}_{k}$ :

$$
\begin{array}{ll}
\dot{\hat{x}}(t)=A \hat{x}(t)+B u(t), \quad\left(\hat{s}_{k} \leq t<t_{k+1}\right) \\
\hat{x}\left(\hat{s}_{k}\right)=x\left(s_{k}\right) & \left(k \in \mathbb{Z}_{+}\right),
\end{array}
$$

where $\hat{x} \in \mathbb{R}^{n}$ is the estimated state. This estimate leads to the discretized extended system in Fig. 2, with state $\xi_{k}$ and input $u_{k}$ given by

$$
\xi_{k}=\left[\begin{array}{c}
x\left(t_{k}\right)-\hat{x}\left(t_{k}\right) \\
\hat{x}\left(t_{k}\right)
\end{array}\right], \quad u_{k}=u\left(t_{k}\right),
$$

respectively, which can be shown to evolve according to

$$
\xi_{k+1}=F_{\Delta_{k}} \xi_{k}+G_{\Delta_{k}} u_{k}, \quad y_{k}=H \xi_{k},
$$

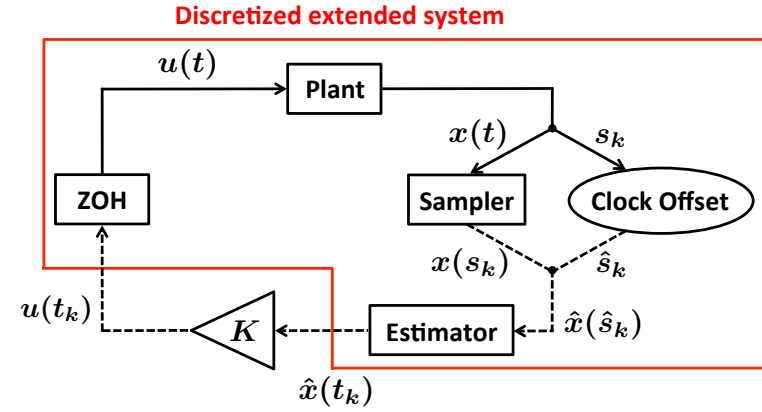

Fig. 2: Closed-loop system with clock offsets

where $p:=e^{A h}, \theta\left(\Delta_{k}\right):=e^{-A \Delta_{k}}-I$, and

$$
\begin{aligned}
F_{\Delta_{k}} & :=\left[\begin{array}{cc}
-p \theta\left(\Delta_{k}\right) & -p \theta\left(\Delta_{k}\right) \\
p\left(I+\theta\left(\Delta_{k}\right)\right) & p\left(I+\theta\left(\Delta_{k}\right)\right)
\end{array}\right] \\
G_{\Delta_{k}} & :=\left[\begin{array}{c}
-p \theta\left(\Delta_{k}\right) A^{-1} B \\
\left(p\left(I+\theta\left(\Delta_{k}\right)\right)-I\right) A^{-1} B
\end{array}\right] \\
H & :=\left[\begin{array}{ll}
0 & I
\end{array}\right] .
\end{aligned}
$$

For the stabilization of the system (II.2), we use a static output feedback $u(t)=u_{k}=K y_{k}=K \hat{x}\left(t_{k}\right)$ for $t \in$ $\left[t_{k}, t_{k+1}\right), k \geq 1$ and $u(t)=u_{0}=0$ for $t \in[0, h)$. The measurement $x\left(s_{0}\right)$ cannot be used for control in $[0, h)$ from Assumption 2.2. We therefore put no control signal in $[0, h)$, which makes the initial condition of the plant's state simple when we study the stability of systems with actuator saturation in Section IV.

The main objective of the present paper is to determine whether, given a feedback gain $K$ and a clock offset range $[\underline{\Delta}, \bar{\Delta}]$, the closed-loop system is stable for every timevarying clock offset sequence $\Delta_{k}$ in $[\underline{\Delta}, \bar{\Delta}]$. We also propose methods to design a feedback gain $K$ that stabilizes the system for every clock offset in a given range.

\section{Stability ANALYSis and STABILIZATION based ON POLYTOPIC OVERAPPROXIMATION}

In this section, we study the stability of the system (II.2) with static feedback control and the design of stabilizing feedback gains, by using the polytopic overapproximation in [2], [14].

\section{A. Stability Analysis}

Define $\Theta$ by $\Theta:=\{\theta(\Delta): \Delta \in[\underline{\underline{\Delta}}, \bar{\Delta}]\}$. Construct matrices $T_{i} \in \mathbb{R}^{n \times n}, U_{i} \in \mathbb{R}^{n \times \phi}$, and $V_{i} \in \mathbb{R}^{\phi \times n}$ overapproximating $\Theta$ as follows:

$\boldsymbol{\Theta} \subset\left\{\sum_{i=1}^{N} \alpha_{i}\left(T_{i}+U_{i} \Phi V_{i}\right): \alpha:=\left\{\alpha_{i}\right\}_{i=1}^{N} \in \mathcal{A}, \Phi \in \mathbf{\Phi}\right\}$,

where $\mathcal{A}$ and $\Phi$ are given by

$$
\begin{aligned}
\mathcal{A}:= & \left\{\left\{\alpha_{i}\right\}_{i=1}^{N}: \sum_{i=1}^{N} \alpha_{i}=1, \alpha_{i} \geq 0 \text { for } i=1, \ldots, N\right\} \\
\boldsymbol{\Phi}:= & \left\{\operatorname{diag}\left(\Phi^{1}, \ldots, \Phi^{L}\right):\right. \\
& \left.\Phi^{j} \in \mathbb{R}^{\phi_{j} \times \phi_{j}},\left\|\Phi^{j}\right\| \leq 1 \text { for } j=1, \ldots, L\right\}
\end{aligned}
$$


for some fixed $\left\{\phi_{i}\right\}_{i=1}^{L}$ satisfying $\phi=\sum_{j=1}^{L} \phi_{j}$. See [2], [14] for a construction method for $T_{i}, U_{i}, V_{i}$, and $\left\{\phi_{i}\right\}_{i=1}^{L}$ satisfying (III.1).

Since $\theta\left(\Delta_{k}\right) \in \boldsymbol{\Theta}$ for every $k \in \mathbb{Z}_{+}$, we have

$$
\theta\left(\Delta_{k}\right)=\sum_{i=1}^{N} \alpha_{k, i}\left(T_{i}+U_{i} \Phi_{k} V_{i}\right)
$$

for appropriately selected $\Phi_{k} \in \boldsymbol{\Phi}$ and $\left\{\alpha_{k, i}\right\}_{i=1}^{N} \in \mathcal{A}$. Using the representation (III.2) for $\theta\left(\Delta_{k}\right)$, we can embed the system (II.2) into the new polytopic system with structured uncertainty given by

$$
\begin{gathered}
\xi_{k+1}= \\
\sum_{i=1}^{N} \alpha_{k, i}\left(\left[\begin{array}{cc}
-p\left(T_{i}+U_{i} \Phi_{k} V_{i}\right) & -p\left(T_{i}+U_{i} \Phi_{k} V_{i}\right) \\
p\left(I+T_{i}+U_{i} \Phi_{k} V_{i}\right) & p\left(I+T_{i}+U_{i} \Phi_{k} V_{i}\right)
\end{array}\right] \xi_{k}\right. \\
\left.+\left[\begin{array}{c}
-p\left(I+T_{i}+U_{i} \Phi_{k} V_{i}\right) A^{-1} B \\
\left(p\left(I+T_{i}+U_{i} \Phi_{k} V_{i}\right)-I\right) A^{-1} B
\end{array}\right] u_{k}\right) \\
y_{k}=\left[\begin{array}{ll}
0 & I
\end{array}\right] \xi_{k} .
\end{gathered}
$$

The system (III.3) with the static feedback controller $u_{k}=$ $K y_{k}$ can be expressed as

$$
\xi_{k+1}=\sum_{i=1}^{N} \alpha_{k, i}\left(A_{c l, i}+B_{c l, i} \Phi_{k} C_{c l, i}\right) \xi_{k}
$$

with $\Phi_{k} \in \boldsymbol{\Phi}$ and $\left\{\alpha_{k, i}\right\}_{i=1}^{N} \in \mathcal{A}$ for all $k \in \mathbb{Z}_{+}$, where

$$
\begin{aligned}
A_{c l, i} & :=\left[\begin{array}{cc}
-p T_{i} & -p T_{i}\left(I+A^{-1} B K\right) \\
p\left(I+T_{i}\right) & p\left(I+T_{i}\right)\left(I+A^{-1} B K\right)-A^{-1} B K
\end{array}\right] \\
B_{c l, i} & :=\left[\begin{array}{c}
-p \\
p
\end{array}\right] U_{i}, \quad C_{c l, i}:=V_{i}\left[\begin{array}{ll}
I & I+A^{-1} B K
\end{array}\right] .
\end{aligned}
$$

Using a parameter-dependent Lyapunov function

$$
V(k, \xi)=\xi^{\top}\left(\sum_{i=1}^{N} \alpha_{k, i} P_{i}\right) \xi,
$$

we can analyze the stability of the derived system (III.4) in terms of LMIs.

Theorem 3.1 ([2], [14]): Let Assumptions 2.1 and 2.2 hold. Define the set of matrices

$$
\begin{array}{r}
\mathcal{R}:=\left\{\operatorname{diag}\left(r_{1} I_{1}, \ldots, r_{L} I_{L}\right) \in \mathbb{R}^{\phi \times \phi}:\right. \\
\left.r_{j}>0 \text { for } j=1, \ldots, L\right\},
\end{array}
$$

where $I_{j}$ is the identity matrix of size $\phi_{j}$. Fix $\gamma \in(0,1]$. If there exist matrices $P_{i}>0$ and $R_{i} \in \mathcal{R}$ for $i=1, \ldots, N$, such that

$$
\left[\begin{array}{cccc}
\gamma P_{i} & 0 & A_{c l, i}^{\top} P_{j} & C_{c l, i}^{\top} R_{i} \\
* & R_{i} & B_{c l, i}^{\top} P_{j} & 0 \\
* & * & P_{j} & 0 \\
* & * & * & R_{i}
\end{array}\right]>0
$$

for all $i, j=1, \ldots, N$, then the closed-loop system (III.4) is exponentially stable with a decay rate less than $\gamma$.

Proof: Only asymptotic stability is discussed in [2], [14], but the extension to exponential stability is easy. We therefore omit the proof.
Remark 3.2: Theorem 3.1 provides a sufficient condition for stability. However, the polytopic overapproximation does not introduce conservatism as follows: If the original system (II.2) is quadratically stable in the sense that there exists a continuous parameter-dependent quadratic Lyapunov function, then this gridding approach guarantees exponential stability with a sufficiently fine grid size with a large $N$; see [2], [14] for details.

In the example below, by using Theorem 3.1, we obtain a range $[\underline{\Delta}, \bar{\Delta}]$ of offsets for which a given feedback system is stable.

Example 3.3 (Vehicle suspension [19]): An active suspension system is given in [19] as follows:

$\frac{d}{d t}\left[\begin{array}{l}x_{1} \\ x_{2} \\ x_{3} \\ x_{4}\end{array}\right]=\left[\begin{array}{cccc}0 & 0 & 1 & -1 \\ 0 & 0 & 0 & 1 \\ -\frac{k_{s}}{m_{s}} & 0 & -\frac{c_{s}}{m_{s}} & \frac{c_{s}}{m_{s}} \\ \frac{k_{s}}{m_{u}} & -\frac{k_{t}}{m_{u}} & \frac{c_{s}}{m_{u}} & \frac{c_{s}+c_{t}}{m_{u}}\end{array}\right]\left[\begin{array}{l}x_{1} \\ x_{2} \\ x_{3} \\ x_{4}\end{array}\right]+\left[\begin{array}{c}0 \\ 0 \\ \frac{1}{m_{s}} \\ -\frac{1}{m_{u}}\end{array}\right] u$,

where $x_{1}, x_{2}, x_{3}$, and $x_{4}$ correspond to the suspension deflection, the tire deflection, the sprung mass speed, and the unsprung mass speed, respectively. Also $m_{s}$ is the sprung mass, which represents the car chassis; $m_{u}$ is the unsprung mass, which represents the wheel assembly; $c_{s}$ and $k_{s}$ are damping and stiffness of the passive suspension system, respectively; $c_{t}$ and $k_{t}$ stand for the damping and compressibility of the pneumatic tire, respectively. Here we omit the input disturbance, which represents the vertical ground velocity of the road profile. These parameters have the following values: $m_{s}=972.2 \mathrm{~kg}, m_{u}=113.6 \mathrm{~kg}$, $c_{s}=1096 \mathrm{Ns} / \mathrm{m}, c_{t}=14.6 \mathrm{Ns} / \mathrm{m}, k_{s}=42719.6 \mathrm{~N} / \mathrm{m}$, and $k_{t}=101115 \mathrm{~N} / \mathrm{m}$. As in [19], we took the feedback control $u=K x$ with

$$
K=-10^{4} \times\left[\begin{array}{llll}
0.3292 & 0.6361 & 1.0125 & 0.0020
\end{array}\right] .
$$

We set the sampling period $h=0.1 \mathrm{sec}$, the decay rate $\gamma=$ 1 , and the number of vertices in the overapproximation $N=$ 26 in (III.1) with equal partitioning. We can conclude from Theorem 3.1 that the closed-loop system is stable whenever the time-varying offset $\Delta_{k}$ belongs to $[-0.0760,0.0818]$. On the other hand, if the clock offset is constant, then the maximum allowable offset interval is $[-0.0792,0.0955]$ for the given gain (III.8), which was obtained by checking the eigenvalue of the closed-loop system for each constant clock offset $\Delta$ and can be regarded as a necessary condition for stability with time-varying offsets.

Fig. 3 shows the relationship between the number of segments $N$ with equal partitioning and the allowable offset interval $[\underline{\Delta}, \bar{\Delta}]$. We observe that as $N$ increases, the numerical result becomes less conservative, but that there is almost no difference on $[\underline{\underline{\Delta}}, \bar{\Delta}]$ between $N=21$ and $N=26$. As stated in Remark 3.2, if $N$ is sufficiently large, then Theorem 3.1 gives the exact offset bound for quadratic stability. Fig. 3 illustrates this fact.

Remark 3.4: We used a static stabilizer here, but we can extend Theorems 3.1 to the case of a general-order stabilizer as follows. 


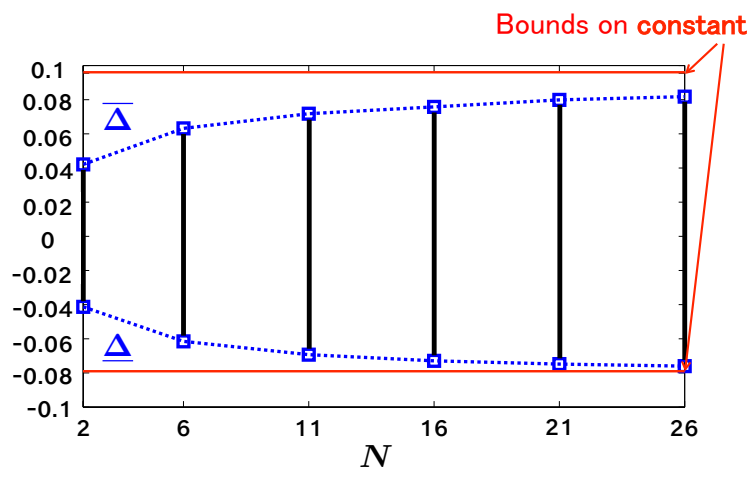

Fig. 3: Number of segments $N$ versus allowable offset interval $[\underline{\Delta}, \bar{\Delta}]$

Let a stabilizable and detectable realization of the dynamic stabilizer be $z_{k+1}=A_{c} z_{k}+B_{c} y_{k}, u_{k}=C_{c} z_{k}+D_{c} y_{k}$. Then the state equation of the closed-loop system is given by

$$
\left[\begin{array}{c}
\xi_{k+1} \\
z_{k+1}
\end{array}\right]=\left[\begin{array}{cc}
F_{\Delta_{k}}+G_{\Delta_{k}} D_{c} H_{\Delta_{k}} & G_{\Delta_{k}} C_{c} \\
B_{c} H_{\Delta_{k}} & A_{c}
\end{array}\right]\left[\begin{array}{l}
\xi_{k} \\
z_{k}
\end{array}\right]
$$

We can rewrite this state equation in the form (III.4) with

$$
\begin{aligned}
A_{c l, i} & =\left[\begin{array}{c|c}
A_{c l, i}^{[1]} & A_{c l, i}^{[2]} \\
\hline A_{c l, i}^{[3]} & A_{c l, i}^{[4]}
\end{array}\right], \quad B_{c l, i}=\left[\begin{array}{c}
-p U_{i} \\
p U_{i} \\
\hline 0
\end{array}\right] \\
C_{c l, i} & =\left[\begin{array}{ll|l}
V_{i} & V_{i}\left(I+A^{-1} B D_{c}\right) \mid V_{i} A^{-1} B C_{c}
\end{array}\right],
\end{aligned}
$$

where

$$
\begin{aligned}
A_{c l, i}^{[1]} & :=\left[\begin{array}{cc}
-p T_{i} & -p T_{i}\left(I+A^{-1} B D_{c}\right) \\
p\left(I+T_{i}\right) & p\left(I+T_{i}\right)\left(I+A^{-1} B D_{c}\right)-A^{-1} B D_{c}
\end{array}\right], \\
A_{c l, i}^{[2]} & :=\left[\begin{array}{c}
-p T_{i} A^{-1} B C_{c} \\
\left(p\left(I+T_{i}\right)-I\right) A^{-1} B C_{c}
\end{array}\right] \\
A_{c l, i}^{[3]} & :=\left[\begin{array}{ll}
0 & B_{c}
\end{array}\right], \quad A_{c l, i}^{[4]}:=A_{c} .
\end{aligned}
$$

\section{B. Stabilization}

We propose two approaches for the design of a stabilizing feedback gain $K$. The main difficulty is the LMI in (III.7) that has multiple product terms $A_{c l, i}^{\top} P_{j}$ and $C_{c l, i}^{\top} R_{i}$, where the unknown $K$ and $P_{j}, R_{i}$ appear multiplied. To circumvent this difficulty, the first approach fixes the positive definite matrices of the quadratic Lyapunov function $V$ in (III.5) as in [20], [21]. The second approach uses the cone complementarity linearization (CCL) algorithm [22].

In the first approach, we use the following partitioned positive definite matrix: Let a positive definite matrix $Q$ be partitioned into

$$
Q=\left[\begin{array}{ll}
Q_{11} & Q_{12} \\
Q_{12}^{\top} & Q_{22}
\end{array}\right]>0
$$

If we define $\Psi:=\left(Q_{12} Q_{22}^{-1}\right)^{\top}, Q_{1}:=Q_{11}-Q_{12} Q_{22}^{-1} Q_{12}^{\top}$, and $Q_{2}:=Q_{22}$, then we can rewrite $Q$ as

$$
Q=\left[\begin{array}{cc}
Q_{1}+\Psi^{\top} Q_{2} \Psi & \Psi^{\top} Q_{2} \\
* & Q_{2}
\end{array}\right]=T_{\Psi} Q_{d} T_{\Psi}^{\top}
$$

where $Q_{d}=\operatorname{diag}\left(Q_{1}, Q_{2}\right)$ and

$$
T_{\Psi}:=\left[\begin{array}{cc}
I & \Psi \\
0 & I
\end{array}\right] .
$$

We see from (III.9) that if $Q>0$, then $Q_{d}$ defined above satisfies $Q_{d}>0$.

Conversely, for every $Q_{d}:=\operatorname{diag}\left(Q_{1}, Q_{2}\right)>0$ and $T_{\Psi}$ defined by (III.10) with an arbitrary matrix $\Psi, Q:=$ $T_{\Psi} Q_{d} T_{\Psi}^{\top}>0$.

The following theorem gives a sufficient condition for the gain synthesis, using the structured positive definite matrix $Q$ in (III.9) with a fixed $\Psi$ :

Theorem 3.5: Let Assumptions 2.1 and 2.2 hold. Define the set $\mathcal{R}$ of matrices as in (III.6). Let $\gamma$ be in $(0,1]$ and fix a matrix $\Psi \in \mathbb{R}^{n \times n}$. If there exist matrices $Q_{i}^{(1)}>0$ and $S_{i} \in \mathcal{R}$ for $i=1, \ldots, N, Q^{(2)}>0$, and $X \in \mathbb{R}^{m \times n}$ such that for all $i, j=1, \ldots, N$,

$$
\left[\begin{array}{cccc}
\gamma Q_{i} & 0 & \Gamma_{A, i}^{\top} & \Gamma_{C, i}^{\top} \\
* & S_{i} & S_{i} B_{c l, i}^{\uparrow} & 0 \\
* & * & Q_{j} & 0 \\
* & * & * & S_{i}
\end{array}\right]>0
$$

where

$$
\begin{aligned}
Q_{i}:= & {\left[\begin{array}{cc}
I & \Psi \\
0 & I
\end{array}\right]\left[\begin{array}{cc}
Q_{i}^{(1)} & 0 \\
0 & Q^{(2)}
\end{array}\right]\left[\begin{array}{ll}
I & \Psi \\
0 & I
\end{array}\right]^{\top} } \\
\Gamma_{A, i}:= & {\left[\begin{array}{cc}
-p T_{i} & -p T_{i} \\
p\left(I+T_{i}\right) & p\left(I+T_{i}\right)
\end{array}\right] Q_{i} } \\
& +\left[\begin{array}{c}
-p T_{i} A^{-1} B \\
\left(p\left(I+T_{i}\right)-I\right) A^{-1} B
\end{array}\right]\left[\begin{array}{ll}
X \Psi^{\top} & X
\end{array}\right] \\
\Gamma_{C, i}:= & {\left[\begin{array}{ll}
V_{i} & V_{i}
\end{array}\right] Q_{i}+V_{i} A_{i}^{-1} B\left[X \Psi^{\top} \quad X\right], }
\end{aligned}
$$

then $K:=X\left(Q^{(2)}\right)^{-1}$ stabilizes the closed-loop system (III.4).

Proof: We aim to transform (III.7) into (III.11) with the structured Lyapunov matrix defined by $Q_{i}$ in (III.12). We see that the LMI in (III.7) is equivalent to

$$
\left[\begin{array}{cccc}
\gamma P_{i}^{-1} & 0 & P_{i}^{-1} A_{c l, i}^{\top} & P_{i}^{-1} C_{c l, i}^{\top} \\
* & R_{i}^{-1} & R_{i}^{-1} B_{c l, i}^{\top} & 0 \\
* & * & P_{j}^{-1} & 0 \\
* & * & * & R_{i}^{-1}
\end{array}\right]>0
$$

by using the congruence transformation $T=$ $\operatorname{diag}\left(P_{i}^{-1}, R_{i}^{-1}, P_{j}^{-1}, R_{i}^{-1}\right)$. Fix the structure of $P_{i}^{-1}=: Q_{i}$ by (III.12) and define $S_{i}:=R_{i}^{-1}$. A routine calculation shows that $A_{c l, i} Q_{i}=\Gamma_{A, i}$ and $C_{c l, i} Q_{i}=\Gamma_{C, i}$ with $X=K Q^{(2)}$, and hence we obtain (III.11).

The second approach is based on the CCL algorithm [22]. The following theorem provides a stability condition in terms of the feedback gain $K$, which can be found using the CCL algorithm:

Theorem 3.6: Let Assumptions 2.1 and 2.2 hold. Define the set $\mathcal{R}$ of matrices as in (III.6). Fix $\gamma \in(0,1]$. If there exist matrices $P_{i}, Q_{i}>0$ and $S_{i} \in \mathcal{R}$ for $i=1, \ldots, N$, and 
$K \in \mathbb{R}^{m \times n}$ such that for all $i, j=1, \ldots, N$,

$$
\left[\begin{array}{cccc}
\gamma P_{i} & 0 & A_{c l, i}^{\top} & C_{c l, i}^{\top} \\
* & S_{i} & S_{i} B_{c l, i}^{\top} & 0 \\
* & * & Q_{j} & 0 \\
* & * & * & S_{i}
\end{array}\right]>0, \quad\left[\begin{array}{cc}
P_{i} & I \\
* & Q_{i}
\end{array}\right] \geq 0,
$$

and $\operatorname{trace}\left(P_{i} Q_{i}\right)=2 n$, then $K$ stabilizes the closed-loop system (III.4).

Proof: For all $P_{i}, Q_{i}>0$ satisfying the second inequality of (III.13), it turns out that trace $\left(P_{i} Q_{i}\right) \geq 2 n$. Furthermore trace $\left(P_{i} Q_{i}\right)=2 n$ if and only if $P_{i} Q_{i}=I$.

Define $S_{i}:=R_{i}^{-1}$. Since $P_{i}=Q_{i}^{-1}$, if we apply the congruence transformation $T=\operatorname{diag}\left(I, S_{i}, Q_{j}, S_{i}\right)$ to (III.7), we see that (III.7) is equivalent to the first condition of (III.13).

Since $\min \left(\operatorname{trace}\left(P_{i} Q_{i}\right)\right)=2 n$ under (III.13) as shown in the proof above, the conditions in Theorem 3.6 are feasible if the problem of minimizing trace $\left(\sum_{i=1}^{N} P_{i} Q_{i}\right)$ under (III.13) has the solution $2 n N$. The CCL algorithm solves this constrained minimization problem. Although the CCL algorithm does not always find the global optimal solution, the non-linear minimization problem is easier to solve than the original non-convex feasibility problem [23].

\section{Systems with Clock Offsets and Actuator SATURATION}

In this section, the results in Theorem 3.1 are extended to the case when the system has both clock offsets and actuator saturation. To this end, we use the regional sector characterization of the saturation nonlinearity in [16], [17], [18, Chapter 3].

Let us consider the system (II.2) with input saturation:

$$
\begin{aligned}
\xi_{k+1} & =F_{\Delta_{k}} \xi_{k}+G_{\Delta_{k}} \sigma_{k} \\
u_{k} & =K_{H} \xi_{k}+L \sigma_{k} \\
\sigma_{k} & =\operatorname{sat}\left(u_{k}\right),
\end{aligned}
$$

with $F_{\Delta_{k}}, G_{\Delta_{k}}$, and $H$ defined by (II.3) and $K_{H}:=(I-$ $L) K H$, where $K$ and $L$ are a control feeback gain and an anti-windup gain, respectively. We obtained $u_{k}=K_{H} \xi_{k}+$ $L \sigma_{k}$ from $u_{k}=K y_{k}+L\left(\sigma_{k}-K y_{k}\right)$.

The function sat $: \mathbb{R}^{m} \rightarrow \mathbb{R}^{m}$ is defined by $\operatorname{sat}(u):=$ $\left[\sigma_{1}(u) \cdots \sigma_{m}(u)\right]$, where $\sigma_{i}$ depends only on the $i$-th input component $u_{i}$ as follows:

$$
\sigma_{i}(u):= \begin{cases}-\bar{u}_{i} & u_{i}<-\bar{u}_{i} \\ u_{i} & -\bar{u}_{i} \leq u_{i} \leq \bar{u}_{i} \\ \bar{u}_{i} & u_{i}>\bar{u}_{i}\end{cases}
$$

with $\bar{u}_{i}>0$.

In this section, we make the following assumption:

Assumption 4.1: The matrix $I-L$ is invertible.

The invertibility of $I-L$ is necessary for the wellposedness of the closed-loop system, because $u_{k}-L \operatorname{sat}\left(u_{k}\right)=K_{H} \xi_{k}$.

We are now ready to state the main result in this section: A sufficient condition for the regional stability of the closedloop system with clock offset and actuator saturation.
Theorem 4.2: Consider the system (IV.1). Let Assumptions 2.1, 2.2, and 4.1 hold. Define the set $\mathcal{R}$ of matrices as in (III.6) and let $e_{l} \in \mathbb{R}^{m}$ be a column vector such that the l-th element is one and the other elements are zero. Fix $\gamma \in(0,1]$ and a positive semidefinite matrix $\Omega \in \mathbb{R}^{n \times n}$. If there exist matrices $Q_{i}>0, S_{i}, \hat{S}_{i} \in \mathcal{R}$, and $Y_{i} \in \mathbb{R}^{m \times 2 n}$ for $i=1, \ldots, N$, and a diagonal matrix $\Lambda_{i}>0$ in $\mathbb{R}^{m \times m}$ such that the following three LMIs hold for all $i, j=1, \ldots, N$ :

$$
\begin{gathered}
{\left[\begin{array}{cccc}
\tilde{Q}_{i} & 0 & {\left[\begin{array}{cc}
Q_{i} & 0 \\
0 & \Lambda
\end{array}\right] \tilde{A}_{c l, i}^{\top}} & {\left[\begin{array}{cc}
Q_{i} & 0 \\
0 & \Lambda
\end{array}\right] \tilde{C}_{c l, i}^{\top}} \\
* & S_{i} & S_{i} \tilde{B}_{c l, i}^{\top} & 0 \\
* & * & Q_{j} & 0 \\
* & * & * & S_{i}
\end{array}\right]>0} \\
{\left[\begin{array}{cc}
Q_{i} & Y_{i}^{\top} e_{l} \\
* & \bar{u}_{i}^{2}
\end{array}\right] \geq 0} \\
{\left[\begin{array}{cccc}
\Omega & 0 & \hat{A}_{c l, i}^{\top} & \hat{C}_{c l, i}^{\top} \\
* & \hat{S}_{i} & \hat{S}_{i} \hat{B}_{c l, i}^{\top} & 0 \\
* & * & Q_{j} & 0 \\
* & * & * & \hat{S}_{i}
\end{array}\right] \geq 0}
\end{gathered}
$$

where

$$
\begin{aligned}
\tilde{K}_{H}:= & K\left[\begin{array}{ll}
0 & I
\end{array}\right], \quad \tilde{L}:=-(I-L)^{-1} L \\
J:= & \tilde{L}-I, \quad \tilde{Q}_{i}:=\left[\begin{array}{cc}
\gamma Q_{i} & -Y_{i}^{\top}-Q_{i} \tilde{K}_{H}^{\top} \\
* & 2 U-\tilde{L} \Lambda-\Lambda \tilde{L}^{\top}
\end{array}\right] \\
\tilde{A}_{c l, i}:= & {\left[\begin{array}{cc}
0 & 0 \\
p & p\left(I+A^{-1} B K\right)-A^{-1} B K \mid(p-I) A^{-1} B J
\end{array}\right] } \\
& +\left[\begin{array}{c}
-p \\
p
\end{array}\right] T_{i}\left[\begin{array}{ll}
I & I+A^{-1} B K \mid A^{-1} B J
\end{array}\right] \\
\tilde{B}_{c l, i}:= & {\left[\begin{array}{c}
-p \\
p
\end{array}\right] U_{i}, \tilde{C}_{c l, i}:=V_{i}\left[\begin{array}{ll}
I+A^{-1} B K \mid A^{-1} B J
\end{array}\right] } \\
\hat{A}_{c l, i}:= & {\left[\begin{array}{c}
-p \\
p
\end{array}\right] T_{i}+\left[\begin{array}{l}
0 \\
p
\end{array}\right], \quad \hat{B}_{c l, i}=\left[\begin{array}{c}
-p \\
p
\end{array}\right] U_{i}, \quad \hat{C}_{c l, i}=V_{i}, }
\end{aligned}
$$

then the system (IV.1) is well posed, and for every initial state $x(0) \in\left\{x \in \mathbb{R}^{n}: x^{\top} \Omega x \leq 1\right\}$, the system (IV.1) with $\hat{x}(0)=0$ is exponentially stable with a decay rate less than $\gamma$

Proof: In order to prove that the Lyapunov function $V$ in (III.5) with $P_{i}>0(i=1, \ldots, N)$ decreases along the trajectory (IV.1), we use the sector characterization of the saturation nonlinearity for stability analysis over a bounded region in [16], [17], [18, Chapter 3]: If the pair $(u, \sigma)$ satisfies $\sigma=\operatorname{sat}(u)$, then for every matrix $M \in \mathbb{R}^{m \times 2 n}$ and every diagonal positive definite matrix $W \in \mathbb{R}^{m \times m}$, we have

$$
\begin{array}{r}
(u, \sigma) \in\left\{(u, \sigma):(\sigma+M \xi)^{\top} W(u-\sigma) \geq 0\right. \\
\text { for all } \xi \text { satisfying } \operatorname{sat}(M \xi)=M \xi\} .
\end{array}
$$

To obtain the LMI (IV.3), we need to change the variable $\sigma=\operatorname{sat}(u)$ to $q:=u-\sigma$, which indicates the deadzone nonlinearity. Then the system (IV.1) is transformed into

$$
\begin{aligned}
\xi_{k+1} & =\tilde{F}_{\Delta_{k}} \xi_{k}+\tilde{G}_{\Delta_{k}} q_{k} \\
u_{k} & =\tilde{K}_{H} \xi_{k}+\tilde{L} q_{k} \\
q_{k} & =u_{k}-\operatorname{sat}\left(u_{k}\right),
\end{aligned}
$$


where $\tilde{K}_{H}$ and $\tilde{L}$ are defined as in the statement of Theorem 4.2 and $\tilde{F}_{\Delta_{k}}:=F_{\Delta_{k}}+G_{\Delta_{k}} K H, \tilde{G}_{\Delta_{k}}:=-G_{\Delta_{k}}((I-$ $\left.L)^{-1} L+I\right)$. Here we used the invertibility of $I-L$ in Assumption 4.1. Under the relationship $q=u-\sigma$, (IV.6) is equivalent to

$$
\begin{aligned}
(u, q) \in\left\{(u, q):(u-q+M \xi)^{\top} W q \geq 0\right. \\
\\
\text { for all } \xi \text { satisfying } \operatorname{sat}(M \xi)=M \xi\} .
\end{aligned}
$$

Using the sector characterization in (IV.8), we show that the Lyapunov function $V$ along the trajectory (IV.7) decreases if the LMI (IV.3) is feasible and if $\xi_{k}$ satisfies $\operatorname{sat}\left(M^{[k]} \xi_{k}\right)=M^{[k]} \xi_{k}$ :

Lemma 4.3: If there exist matrices $Q_{i}>0, S_{i} \in \mathcal{R}$, $Y_{i} \in \mathbb{R}^{m \times 2 n}$ for $i=1, \ldots, N$, and a diagonal matrix $\Lambda>0$ in $\mathbb{R}^{m \times m}$ such that the LMI (IV.3) holds for every $i, j=1, \ldots, N$, then the system (IV.7) is well posed and the following statement on the Lyapunov function $V$ in (III.5) along the trajectory (IV.7) is true:

$$
\begin{gathered}
\left(u_{k}-q_{k}+M^{[k]} \xi_{k}\right) W q_{k} \geq 0 \text { for all }\left(\xi_{k}, q_{k}\right) \neq 0 \\
\Rightarrow \quad V\left(k+1, \xi_{k+1}\right)-\gamma V\left(k, \xi_{k}\right)<0,
\end{gathered}
$$

where we define $P_{i}:=Q_{i}^{-1}, W:=\Lambda^{-1}, M_{i}:=Y_{i} Q_{i}^{-1}$, and using $\left\{\alpha_{k, i}\right\}_{i=1}^{N}$ in (III.2), $M^{[k]}:=\sum_{i=1}^{N} \alpha_{k, i} M_{i}$.

Proof: See Section IV. A.

Furthermore, we see from the next lemma that the remaining condition $\operatorname{sat}\left(M^{[k]} \xi_{k}\right)=M^{[k]} \xi_{k}$ for the decrease of the Lyapunov function $V$ holds if $V\left(k, \xi_{k}\right) \leq 1$ and the LMI (IV.4) is feasible. The condition $V\left(1, \xi_{1}\right) \leq 1$ is implied by $x_{0}^{\top} \Omega x_{0} \leq 1$ on the initial state $x_{0}$ and the LMI (IV.5).

Lemma 4.4: Consider the system (IV.7). Suppose that $x_{0}^{\top} \Omega x_{0} \leq 1$ and $\hat{x}(0)=0$. If there exist $Q_{i}>0$ and $\hat{S}_{i} \in \mathcal{R}$ for $i=1, \ldots, N$, such that the LMI (IV.5) holds for every $i, j=1, \ldots, N$, then $\xi_{1}$ satisfies $V\left(1, \xi_{1}\right) \leq 1$ in (III.5) with $P_{i}=Q_{i}^{-1}$. Furthermore, if such $Q_{i}$ satisfies the LMI (IV.4) with some matrix $Y_{i} \in \mathbb{R}^{m \times 2 n}$ for $i=1, \ldots, N$, then $V\left(k, \xi_{k}\right) \leq 1$ implies $\operatorname{sat}\left(M^{[k]} \xi_{k}\right)=M^{[k]} \xi_{k}$ for every $k \in \mathbb{N}$, where $M^{[k]}$ is defined as in Lemma 4.3.

Proof: See Section IV. B.

Since the well-posedness of the systems (IV.7) and (IV.1) have been proved in Lemma 4.3, we only need to show that exponential stability follows from Lemmas 4.3 and 4.4.

Since $\operatorname{sat}\left(M^{[1]} \xi_{1}\right)=M^{[1]} \xi_{1}$ by Lemma 4.4 , it follows that $\left(u_{1}, q_{1}\right)$ in (IV.7) satisfies $\left(u_{1}-q_{1}+M^{[1]} \xi_{1}\right)^{\top} W q_{1} \geq 0$ for every diagonal positive definite matrix $W \in \mathbb{R}^{m \times m}$. If we use $W:=\Lambda^{-1}$, then Lemma 4.3 shows that $V\left(2, \xi_{2}\right) \leq$ $\gamma V\left(1, \xi_{1}\right) \leq 1$, and hence the second statement of Lemma 4.4 gives $\operatorname{sat}\left(M^{[2]} \xi_{2}\right)=M^{[2]} \xi_{2}$. Continuing in this way, we achieve the exponential decrease of the Lyapunov function $V\left(k, \xi_{k}\right)$. Since $P_{i}>0$ for every $i=1, \ldots, N$, it follows that $V\left(k, \xi_{k}\right) \geq \epsilon\left\|\xi_{k}\right\|^{2}$ for some $\epsilon>0$. Thus $\xi_{k}$ converges to the origin exponentially.

Using Theorem 4.2, the following example shows how the offset interval $[\underline{\Delta}, \bar{\Delta}]$ varies with the saturation limit $\bar{u}$ :

Example 4.5 (Aircraft [24]): Let us consider the longitudinal dynamics of the TRANS3 aircraft in [24]. The aircraft

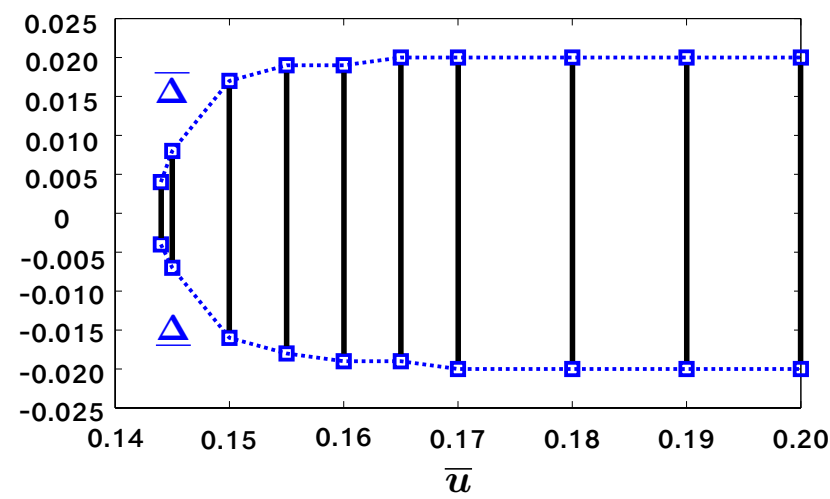

Fig. 4: Allowable offset interval $[\underline{\underline{\Delta}}, \bar{\Delta}]$ and saturation limit $\bar{u}$ versus

model is given by $\dot{x}=A x+B u$, where

$$
A:=\left[\begin{array}{cccc}
0 & 14.3877 & 0 & -31.5311 \\
-0.0012 & -0.4217 & 1 & -0.0284 \\
0.0002 & -0.3816 & -0.4658 & 0 \\
0 & 0 & 1 & 0
\end{array}\right],
$$

$B:=\left[\begin{array}{llll}4.526 & -0.0337 & -1.4566 & 0\end{array}\right]^{\top}$, and $\left[\begin{array}{lll}x_{1} & x_{2} & x_{3} \\ x_{4}\end{array}\right]:=$ $x^{\top}$ are the velocity, the angle of attack, the pitch rate, and the Euler angle rotation of the aircraft about the inertial $y$-axis respectively, and $u$ is the elevator input.

Let the sampling period be $h=0.02 \mathrm{sec}$ and the feedback gain $K$ be the linear quadratic regular for $(A, B)$ with state weight $I$ and input weight 600 . In addition, let the antiwindup gain $L$ be $L=0$. Here we set the matrix $\Omega=$ $\operatorname{diag}(0.05,20,20,20)$ for the initial condition and the decay rate $\gamma=1$. We used $N \leq 5$ in the overapproximation (III.1) with equal partitioning. We note that in this example, an increase in $N$ did not make the analysis by Theorem 4.2 less conservative since $h$ is small. For small values of $h, U_{i}$ in (III.1) and hence $\tilde{B}_{c l, i}, \hat{B}_{c l, i}$ in Theorem 4.2 become small as well as the case of a large $N$.

Fig. 4 shows the allowable offset interval $[\underline{\Delta}, \bar{\Delta}]$ obtained by Theorem 4.2 for each saturation limit $\bar{u}$. We see that if $\bar{u} \geq 0.170$, then the saturation does not affect the allowable offset. However, we have almost no margin on clock offsets for $\bar{u} \leq 0.144$. This is because the plant has two unstable poles $0.0212 \pm j 0.1670$ and the closed-loop system easily becomes unstable for a small saturation limit $\bar{u}$.

Remark 4.6: (1) If we fix the anti-windup gain $L$, then the synthesis technique in Theorem 3.5 can be used in the context of Theorem 4.2 to compute a stabilizing feedback gain $K$.

(2) As in Remark 3.4, we can generalize the result in this section to the case of the following general-order stabilizer:

$$
\begin{aligned}
z_{k+1} & =A_{c} z_{k}+B_{c} y_{k}+L_{1}\left(\sigma_{k}-C_{c} z_{k}-D_{c} y_{k}\right) \\
u_{k} & =C_{c} z_{k}+D_{c} y_{k}+L_{2}\left(\sigma_{k}-C_{c} z_{k}-D_{c} y_{k}\right) \\
\sigma_{k} & =\operatorname{sat}\left(u_{k}\right) .
\end{aligned}
$$


In this case, the close-loop system is given by

$$
\begin{aligned}
{\left[\begin{array}{c}
\xi_{k+1} \\
z_{k+1}
\end{array}\right] } & =\left[\begin{array}{cc}
F_{\Delta_{k}} & 0 \\
\left(B_{c}-L_{1} D_{c}\right) H & A_{c}-L_{1} C_{c}
\end{array}\right]\left[\begin{array}{l}
\xi_{k} \\
z_{k}
\end{array}\right]+\left[\begin{array}{c}
G_{\Delta_{k}} \\
L_{1}
\end{array}\right] \sigma_{k} \\
u_{k} & =\left(I-L_{2}\right)\left[\begin{array}{ll}
D_{c} H & C_{c}
\end{array}\right]\left[\begin{array}{l}
\xi_{k} \\
z_{k}
\end{array}\right]+L_{2} \sigma_{k} \\
\sigma_{k} & =\operatorname{sat}\left(u_{k}\right),
\end{aligned}
$$

which is the same form as in (IV.1). Thus we can apply the approach in this section to (IV.10).

\section{A. Proof of Lemma 4.3}

Define $P^{[k]}:=\sum_{i=1}^{N} \alpha_{k, i} P_{i}$. By the $\mathrm{S}$-procedure, in order to guarantee (IV.9), it is enough to check that for all $(\xi, q) \neq$ 0 ,

$$
\begin{aligned}
& {\left[\begin{array}{c}
\xi \\
q
\end{array}\right]^{\top}\left[\begin{array}{c}
\tilde{F}_{\Delta_{k}}^{\top} \\
\tilde{G}_{\Delta_{k}}^{\top}
\end{array}\right] P^{[k+1]}\left[\begin{array}{ll}
\tilde{F}_{\Delta_{k}} & \tilde{G}_{\Delta_{k}}
\end{array}\right]\left[\begin{array}{c}
\xi \\
q
\end{array}\right]-\xi^{\top} \gamma P^{[k]} \xi} \\
& \quad+2\left(M^{[k]} \xi_{k}+\tilde{K}_{H} \xi_{k}+\tilde{L} q_{k}-q_{k}\right)^{\top} W q_{k}<0,
\end{aligned}
$$

that is,

$$
\left[\begin{array}{c}
\tilde{F}_{\Delta_{k}}^{\top} \\
\tilde{G}_{\Delta_{k}}^{\top^{\top}}
\end{array}\right] P^{[k+1]}\left[\begin{array}{ll}
\tilde{F}_{\Delta_{k}} & \tilde{G}_{\Delta_{k}}
\end{array}\right]-\tilde{P}^{[k]}<0,
$$

where

$$
\tilde{P}^{[k]}:=\left[\begin{array}{cc}
\gamma P^{[k]} & -\left(M^{[k]}+\tilde{K}_{H}\right)^{\top} W \\
* & 2 W-W \tilde{L}-\tilde{L}^{\top} W
\end{array}\right] .
$$

Since $P^{[k+1]}>0$, by the Schur complement, we have that (IV.9) is equivalent to

$$
\left[\begin{array}{cc}
\tilde{P}^{[k]} & {\left[\begin{array}{c}
\tilde{F}_{\Delta_{k}}^{\top} \\
\tilde{G}_{\Delta_{k}}^{\top^{[k+1]}}
\end{array}\right] P^{[k+1]}} \\
* & P^{[k+1]}
\end{array}\right]>0 .
$$

Also (II.3) and (III.1) give

$$
\begin{aligned}
{\left[\begin{array}{ll}
\tilde{F}_{\Delta_{k}} & \tilde{G}_{\Delta_{k}}
\end{array}\right] } & =\left[\begin{array}{ll}
F_{\Delta_{k}}+G_{\Delta_{k}} K H & G_{\Delta_{k}} J
\end{array}\right] \\
& =\sum_{i=1}^{N} \alpha_{k, i}\left(\tilde{A}_{c l, i}+\tilde{B}_{c l, i} \Phi_{k} \tilde{C}_{c l, i}\right),
\end{aligned}
$$

for some $\Phi_{k} \in \boldsymbol{\Phi}$, where $\tilde{A}_{c l, i}, \tilde{B}_{c l, i}$, and $\tilde{C}_{c l, i}$ are defined as in the statement of Theorem 4.2. It follows from the property

$$
\sum_{i=1}^{N} \alpha_{k, i}=1, \quad \alpha_{k, i} \geq 0
$$

that (IV.13) holds for all $\left\{\alpha_{k, i}\right\}_{i=1}^{N} \in \mathcal{A}$ if and only if we have

$$
\left[\begin{array}{cc}
\tilde{P}_{i} & \left(\tilde{A}_{c l, i}+\tilde{B}_{c l, i} \Phi_{k} \tilde{C}_{c l, i}\right)^{\top} P^{[k+1]} \\
* & P^{[k+1]}
\end{array}\right]>0
$$

for all $i=1, \ldots, N$, where $\tilde{P}_{i}$ is defined by (IV.12) with $P_{i}, M_{i}$ in place of $P^{[k]}, M^{[k]}$. Similarly, using the property (IV.14) for $\left\{\alpha_{k+1, i}\right\}_{i=1}^{N}$ again, we show that (IV.15) is satisfied for all $\left\{\alpha_{k+1, i}\right\}_{i=1}^{N} \in \mathcal{A}$ if and only if for all $i, j=1, \ldots, N$,

$$
\left[\begin{array}{cc}
\tilde{P}_{i} & \left(\tilde{A}_{c l, i}+\tilde{B}_{c l, i} \Phi_{k} \tilde{C}_{c l, i}\right)^{\top} P_{j} \\
* & P_{j}
\end{array}\right]>0 .
$$

As shown in [15], [25], since $R-\Phi^{\top} R \Phi \geq 0$ for all $\Phi \in \boldsymbol{\Phi}$ and $R \in \mathcal{R}$, it follows that (IV.16) holds for all $\Phi_{k} \in \boldsymbol{\Phi}$ if

$$
\begin{aligned}
& {\left[\begin{array}{cc}
\tilde{P}_{i}-\tilde{C}_{i}^{\top}\left(R_{i}-\Phi_{k}^{\top} R_{i} \Phi_{k}\right) \tilde{C}_{i} & \left(\tilde{A}_{c l, i}+\tilde{B}_{c l, i} \Phi_{k} \tilde{C}_{c l, i}\right)^{\top} P_{j} \\
* & P_{j}
\end{array}\right]} \\
& =\Pi^{\top}\left[\begin{array}{cccc}
\tilde{P}_{i} & 0 & \tilde{A}_{c l, i}^{\top} P_{j} & \tilde{C}_{c l, i}^{\top} R_{i} \\
* & R_{i} & \tilde{B}_{c l, i}^{\top} P_{j} & 0 \\
* & * & P_{j} & 0 \\
* & * & * & R_{i}
\end{array}\right] \Pi>0,
\end{aligned}
$$

where

$$
\Pi:=\left[\begin{array}{cc}
I & 0 \\
\Phi_{k} \tilde{C}_{c l, i} & 0 \\
0 & I \\
-\tilde{C}_{c l, i} & 0
\end{array}\right] .
$$

Since $\Pi$ is full column rank, (IV.17) holds if

$$
\left[\begin{array}{cccc}
\tilde{P}_{i} & 0 & \tilde{A}_{c l, i}^{\top} P_{j} & \tilde{C}_{c l, i}^{\top} R_{i} \\
* & R_{i} & \tilde{B}_{c l, i}^{\top} P_{j} & 0 \\
* & * & P_{j} & 0 \\
* & * & * & R_{i}
\end{array}\right]>0 .
$$

Since there is a term $M_{i}^{\top} W$ in $\tilde{P}_{i}$, the condition (IV.18) is not an LMI. However, we can transform (IV.18) into an LMI condition. Define $Q_{i}:=P_{i}^{-1}, S_{i}:=R_{i}, \Lambda:=W^{-1}$, and $Y_{i}:=M_{i} Q_{i}$. Using the congruence transformation $T_{1}=$ $\operatorname{diag}\left(Q_{i}, \Lambda, S_{i}, Q_{j}, S_{i}\right)$, we can show that (IV.18) holds if and only if (IV.3) is feasible.

The well-posedness of the closed-loop system, that is, the invertibility of the function $I-(I-\tilde{L} \operatorname{sat}(\bullet))$ is achieved from $2 W-W \tilde{L}-\tilde{L}^{\top} W>0$ in (IV.16) by Proposition 1 of [26].

\section{B. Proof of Lemma 4.4}

We first prove that if the LMI (IV.5) is feasible, then $V\left(1, \xi_{1}\right) \leq 1$ holds for all the initial state $\xi(0)=$ $\left[x(0)^{\top} \hat{x}(0)^{\top}\right]^{\top}$ satisfying $x(0)^{\top} \Omega x(0) \leq 1$ and $\hat{x}(0)=0$.

Since we have $u(t)=0$ for $t \in[0, h)$ from Assumption 4.1 , if $\hat{x}(0)=0$, then $\xi_{1}=\Upsilon\left(\Delta_{0}\right) x(0)$, where

$$
\Upsilon\left(\Delta_{0}\right):=\left[\begin{array}{c}
e^{A h}\left(I-e^{-A \Delta_{0}}\right) \\
e^{A\left(h-\Delta_{0}\right)}
\end{array}\right]=\left[\begin{array}{c}
-p \theta\left(\Delta_{0}\right) \\
p\left(I+\theta\left(\Delta_{0}\right)\right)
\end{array}\right] .
$$

In order to prove that $x(0)^{\top} \Omega x(0) \leq 1$ implies $V\left(1, \xi_{1}\right) \leq 1$, it suffices to show that $x(0)^{\top} \Omega x(0) \geq V\left(1, \xi_{1}\right)$, namely, $P_{j}>0, j=1, \ldots, N$, satisfies

$$
\Omega-\Upsilon\left(\Delta_{0}\right)^{\top} P_{j} \Upsilon\left(\Delta_{0}\right) \geq 0
$$

for all $j=1, \ldots, N$ and for all $\Delta_{0} \in[\underline{\Delta}, \bar{\Delta}]$. Here we used the property (IV.14) for $\left\{\alpha_{1, i}\right\}_{i=1}^{N} \in \mathcal{A}$.

A sufficient condition for (IV.19) to hold can be obtained in the same way as (IV.11). In fact, $\Upsilon\left(\Delta_{0}\right)$ is given by

$$
\Upsilon\left(\Delta_{0}\right)=\sum_{i=1}^{N} \alpha_{0, i}\left(\hat{A}_{c l, i}+\hat{B}_{c l, i} \Phi_{0} \hat{C}_{c l, i}\right)
$$

for some $\Phi_{0} \in \boldsymbol{\Phi}$, where $\hat{A}_{c l, i}, \hat{B}_{c l, i}$, and $\hat{C}_{c l, i}$ are defined as in the statement of Theorem 4.2. It follows from the Schur 
complement and the property (IV.14) for $\left\{\alpha_{0, i}\right\}_{i=1}^{N} \in \mathcal{A}$ that (IV.19) holds for all $\Delta_{0} \in[\underline{\Delta}, \bar{\Delta}]$ if

$$
\left[\begin{array}{cc}
\Omega & \left(\hat{A}_{c l, i}+\hat{B}_{c l, i} \Phi_{0} \hat{C}_{c l, i}\right)^{\top} P_{j} \\
* & P_{j}
\end{array}\right] \geq 0
$$

for all $i=1, \ldots, N$ and $\Phi_{0} \in \boldsymbol{\Phi}$. Moreover, with the transformation similar to that in (IV.17), we have that (IV.20) holds for all $\Phi_{0} \in \boldsymbol{\Phi}$ if there exists $\hat{R}_{i} \in \mathcal{R}$ such that

$$
\left[\begin{array}{cccc}
\Omega & 0 & \hat{A}_{c l, i}^{\top} P_{j} & \hat{C}_{c l, i}^{\top} \hat{R}_{i} \\
* & \hat{R}_{i} & \hat{B}_{c l, i}^{\top} P_{j} & 0 \\
* & * & P_{j} & 0 \\
* & * & * & \hat{R}_{i}
\end{array}\right] \geq 0 .
$$

For the consistency of the variables in the LMI (IV.3), we define $Q_{i}:=P_{i}^{-1}$ and $\hat{S}_{i}:=\hat{R}_{i}^{-1}$ and apply the congruence transformation $T_{2}=\operatorname{diag}\left(I, \hat{S}_{i}, Q_{j}, \hat{S}_{i}\right)$ to (IV.21), which gives (IV.5).

Let us next prove that $V\left(k, \xi_{k}\right) \leq 1$ implies $\operatorname{sat}\left(M^{[k]} \xi_{k}\right)=M^{[k]} \xi_{k}$, To this end, it is enough to show that

$$
\begin{array}{r}
\frac{1}{\bar{u}_{l}^{2}} \xi^{\top}\left(\sum_{i=1}^{N} \alpha_{k, i} M_{i}^{(l)}\right)^{\top}\left(\sum_{i=1}^{N} \alpha_{k, i} M_{i}^{(l)}\right) \xi \\
\leq V(k, \xi)=\xi^{\top}\left(\sum_{i=1}^{N} \alpha_{k, i} P_{i}\right) \xi
\end{array}
$$

for all $l=1, \ldots, m$, where $M_{i}^{(l)}$ denotes the $l$-th row of $M_{i}$. Using the Schur complement, we see from the property (IV.14) for $\left\{\alpha_{k, i}\right\}_{i=1}^{N}$ that (IV.22) holds for all $\left\{\alpha_{k, i}\right\}_{i=1}^{N} \in \mathcal{A}$ if and only if

$$
\left[\begin{array}{cc}
P_{i} & \left(M_{i}^{(l)}\right)^{\top} \\
* & \bar{u}_{l}^{2}
\end{array}\right] \geq 0
$$

for all $i=1, \ldots, N$ and $l=1, \ldots, m$. For the consistency of the variables in the LMI (IV.3), we use the congruence transformation $T_{3}=\operatorname{diag}\left(Q_{i}, I\right)$, which shows that (IV.23) is equivalent to (IV.4)

\section{CONCLUding Remarks}

We have studied the stability analysis and the stabilization problem for systems with time-varying clock offsets. We have handled the parameter uncertainty caused by clock offsets with a polytopic overapproximation, and have derived a sufficient condition for stability. Two methods for the design of stabilizing feedback gains have been proposed, based on fixing the structure of the matrices in the quadratic Lyapunov function and on the CCL algorithm. Using the sector characterization of the saturation nonlinearity, we have also extended this overapproximation approach to the analysis of regional stability for systems with actuator saturation in addition to clock offsets. The design of dynamic feedback controllers and anti-windup gains is left as a topic for future research.

\section{REFERENCES}

[1] J. P. Hespanha, P. Naghshtabrizi, and Y. Xu, "A survey of recent results in networked control systems," Proc. IEEE, vol. 95, pp. 138-162, 2007.

[2] W. P. M. H. Heemels, N. van de Wouw, R. H. Gielen, M. C. F. Donkers, L. Hetel, S. Olaru, M. Lazar, J. Daafouz, and S. Niculescu, "Comparison of overapproximation methods for stability analysis of networked control systems," in Proc. HSCC'10, 2010.

[3] L. Zhang, H. Gao, and O. Kaynak, "Network-induced constraints in networked control systems-A survey," IEEE Trans. Ind. Inform, vol. 9, pp. 406-416, 2013.

[4] S. Graham and P. R. Kumar, "Time in general-purpose control systems: The Control Time Protocol and an experimental evaluation," in Proc. 43rd IEEE CDC, 2004.

[5] Y. Yang, Y. Wang, and S.-H. Yang, "Design of a networked control system with random transmission delay and uncertain process parameters," Int. J. Systems Science, vol. 39, pp. 1065-1074, 2008.

[6] N. M. Freris, S. R. Graham, and P. R. Kumar, "Fundamental limits on synchronizing clocks over network," IEEE Trans. Automat. Control, vol. 56, pp. 1352-1364, 2011.

[7] S. M. LaValle and M. B. Egerstedt, "On time: Clocks, Chronometers, and open-loop control," in Proc. 46th CDC, 2007.

[8] A. Lamperski and N. J. Cowan, "Optimal control with noisy time," Submitted to IEEE Trans. Automat. Control, 2013. [Online]. Available: http://arxiv.org/abs/1401.0202

[9] R. Singh and V. Gupta, "On LQR control with asynchronous clocks," in Proc. 50th CDC, 2011.

[10] M. Wakaiki, K. Okano, and J. P. Hespanha, "Stabilization of networked control systems with clock offset," To appear in Proc. ACC'15.

[11] K. Okano, M. Wakaiki, and J. P. Hespanha, "Real-time control under clock offsets between sensors and controllers," To appear in Proc. HSCC'15.

[12] E. Fridman, A. Seuret, and J.-P. Richard, "Robust sampled-data stabilization of linear systems: An input delay approach," Automatica, vol. 40, pp. 1441-1446, 2004.

[13] E. Fridman and M. Dambrine, "Control under quantization, saturation and delay: An LMI approach," Automatica, vol. 45, pp. 2258-2264, 2009.

[14] M. C. F. Donkers, W. P. M. H. Heemels, N. van de Wouw, and L. Hetel, "Stability analysis of networked control systems using a switched linear systems approach," IEEE Trans. Automat. Control, vol. 56, pp. 2101-2115, 2011.

[15] S. J. L. M. van Loon, M. C. F. Donkers, N. van de Wouw, and W. P. M. H. Heemels, "Stability analysis of networked and quantized linear control systems," Nonlinear Anal.: Hybrid Systems, vol. 10, pp. 111$125,2013$.

[16] T. Hu, A. R. Teel, and L. Zaccarian, "Anti-windup synthesis for linear control systems with input saturation: Achieving regional, nonlinear performance," Automatica, vol. 44, pp. 512-519, 2008.

[17] M. Massimetti, L. Zaccarian, T. Hu, and A. R. Teel, "Linear discretetime global and regional anti-windup: an LMI approach," Int. J. Control, vol. 82, pp. 2179-2192, 2009.

[18] L. Zaccarian and A. R. Teel, Modern Anti-Winndup Synthesis: Control Augmentation for Actuator Saturation. Princeton, NJ: Princeton Univ. Press, 2011.

[19] H. Du and N. Zhang, " $H_{\infty}$ control of active vehicle suspensions with actuator time delay," J. Sound Vib., vol. 301, pp. 236-252, 2007.

[20] E. Prempain and I. Postlethwaite, "Static output feedback stabilization with $\mathcal{H}_{\infty}$ performance for a class of plants," Systems Control Lett., vol. 43, pp. 159-166, 2001.

[21] G. I. Bara and M. Boutayeb, "Static output feedback stabilization with $\mathcal{H}_{\infty}$ performance for linear discrete-time systems," IEEE Trans. Automat. Control, vol. 50, pp. 250-254, 2005.

[22] L. E. Ghaoui, F. Oustry, and M. AitRami, "A cone complementarity linearization algorithm for static output-feedback and related problems," IEEE Trans. Automat. Control, vol. 42, pp. 1171-1176, 1997.

[23] M. C. de Oliveria and J. C. Geromel, "Numerical comparison of output feedback design methods," in Proc. ACC'97, 1997.

[24] T. Hu and Z. Lin, Control Systems with Actuator Saturation: Analysis and Design. Boston, MA: Birkhäuser, 2001.

[25] M. C. F. Donkers, "Networked and Event-Triggered Control Systems," Ph.D. dissertation, Eindhoven Univ. of Technol., 2011.

[26] L. Zaccarian and A. R. Teel, "A common framework for anti-windup, bumpless transfer and reliable designs," Automatica, vol. 38, pp. 17351744, 2002. 